\title{
Fatty Acid Enhancement of the Quantum Yield for the Formation of Lumirubin from Bilirubin Bound to Human Albumin'
}

\author{
VINAY MALHOTRA, JORDAN W. GREENBERG, LAURIE L. DUNN, AND JOHN F. ENNEVER \\ Department of Pediatrics, Rainbow Babies and Childrens Hospital, Case Western Reserve University School of \\ Medicine, Cleveland, Ohio 44106
}

\begin{abstract}
During phototherapy for neonatal jaundice, bilirubin is converted into a number of different polar photoproducts. Because fatty acids are bound to human albumin in vivo and have been shown to affect the binding of bilirubin to albumin, we examined the effect of various fatty acids on the photochemistry of albumin-bound bilirubin. Fatty acids of carbon chain length of 10 or more were found to increase by as much as 3 -fold the quantum yield for formation of lumirubin, an intramolecularly cyclized isomer of bilirubin. The binding of these same fatty acids was found to affect visible absorption and circular dichroic spectra of the bound bilirubin, a finding previously interpreted to be the result of a conformational change in the bilirubin. The increased quantum yield for the formation of lumirubin appears to be the result of an allosterically induced conformational change in bilirubin which is bound to albumin. (Pediatr Res 21: 530-533, 1987)
\end{abstract}

The use of light in the treatment of neonatal jaundice was first described nearly $30 \mathrm{yr}$ ago (1). While little has changed over the past three decades in the way in which phototherapy is administered to jaundiced infants, our understanding of how phototherapy works has been revised and refined many times. Recent evidence from a number of clinical studies indicates that the elimination of bilirubin during phototherapy is principally the result of the formation and excretion of a polar structural isomer of hilirubin, called lumirubin (2-5). The importance of lumiruuin to pigment elimination during phototherapy is surprising because in vitro experiments have shown that the rate of lumirubin formation is slow; however, these measurements have been performed using bilirubin bound to human serum albumin in the absence of fatty acids, which are bound to albumin in vivo $(6,7)$. We report herein that the binding of long-chain fatty acids to bilirubin-albumin complexes in vitro markedly enhances the rate of formation of lumirubin.

\section{METHODS}

All work with bilirubin was performed under photographic safe lights. Crystalline analytical grade bilirubin (Serva Fine Biochemicals, Garden City Park, NY), purified as previously described (8), was dissolved in $0.1 \mathrm{M} \mathrm{NaOH}$ and added to a final concentration of $30 \mu \mathrm{M}$ to a solution of $60 \mu \mathrm{M}$ human albumin

Received October 20, 1986; accepted December 24, 1986.

Correspondence and reprint requests John F. Ennever Ph.D., M.D., Department of Pediatrics, Rainbow Babies and Childrens Hospital, 2101 Adelbert Road, Cleveland, $\mathrm{OH} 44106$.

Supported by the U.S. Public Health Service Grants DK-36517 and CA-23692.

${ }^{1}$ Presented in part at the Annual Meeting of the Society for Pediatric Research, May 1986. (essentially fatty acid free, Sigma Chemical, St. Louis, MO), 0.15 $\mathrm{M} \mathrm{NaCl}, 1 \mathrm{mM} \mathrm{Na} 2$ EDTA, $0.05 \mathrm{M}$ HEPES (N-2-hydroxyethylpiperazine- $\mathrm{N}^{\prime}$-2-ethanesulfonic acid), $\mathrm{pH}$ 7.4. Hexanoic, octanoic, decanoic, lauric, myristic, and stearic acids were obtained from Aldrich Chemical Co. (Milwaukee, WI); palmitic, oleic, and linoleic acids were obtained from Sigma Chemical Co. All were free acids of the highest purity available. The fatty acids were dissolved in methanol containing equimolar $\mathrm{NaOH}$ and aliquots were dried under Ar to a film on glass tubes (9). The bilirubin-albumin solution was added and the solutions were mixed either by vortexing or stirring until optically clear. Three concentrations of fatty acids were studied: 1.0,2.5, and 5.0 equivalents per mole of albumin. Three $\mathrm{ml}$ of each solution was placed in a polystyrene cuvette and sealéd. The cuvettes were placed on the perimeter of a turntable in holders which masked all but the front surface. The samples were irradiated for 30 min with white light from a bank of eight Duro-Test Vita Lites, while the turntable was rotated at $50 \mathrm{rpm}$, ensuring that both the fatty acid-containing and reference (no fatty acid) solutions received equal incident irradiation. Under the conditions used, approximately $6 \%$ of the bilirubin in the reference solution (with no fatty acid) was converted to lumirubin. After the irradiation, the samples were analyzed by high-pressure liquid chromatography as previously described (10). The amount of lumirubin formed in each sample, determined from the integrated area under the lumirubin peak on the chromatogram, was divided by the amount of lumirubin formed in simultaneously irradiated reference solutions.

Absorbance and circular dichroism spectra were obtained on unirradiated samples in $1-\mathrm{cm}$ quartz cuvettes. The absorbance spectra were measured in a Cary 210 dual-beam spectrophotometer. Circular dichroism spectra were measured in a Jasco model J-40A spectrometer, which was calibrated with (+)-10-camphor sulfonic acid.

\section{RESULTS}

For each fatty acid studied, three measurements were made to determine the effects of binding of the acid to bilirubin:albumin complexes: the rate of lumirubin formation, the visible absorption spectrum, and the circular dichroism spectrum. The rate of lumirubin formation from bilirubin bound to human albumin showed a progressive increase with the addition of up to five equivalents of fatty acid of carbon chain length of 10 or more (Table 1). Above five equivalents of fatty acids, further increases in the rate of lumirubin production were seen; however, because of the displacement of bilirubin from albumin at these higher concentrations of fatty acids, the results were variable (results not shown). Hexanoic and octanoic acids, unlike the longer chain fatty acids, produced only a slight increase in the rate of lumirubin production which did not show a trend with concentration (Table 1). 
FATTY ACID EFFECT ON LUMIRUBIN FORMATION

Table 1. Effect of fatty acids on rate of lumirubin formation*

\begin{tabular}{|c|c|c|c|c|c|c|c|c|c|}
\hline $\begin{array}{c}\text { Fatty } \\
\text { acid:HSA } \\
\text { molar ratio }\end{array}$ & $\begin{array}{l}\text { Hexanoic } \\
\text { C-6 }\end{array}$ & $\begin{array}{l}\text { Octanoic } \\
\text { C-8 }\end{array}$ & $\begin{array}{c}\text { Decanoic } \\
\text { C-10 }\end{array}$ & $\begin{array}{c}\text { Lauric } \\
\text { C-12 }\end{array}$ & $\begin{array}{c}\text { Myristic } \\
\text { C-14 }\end{array}$ & $\begin{array}{c}\text { Palmitic } \\
\text { C-16 }\end{array}$ & $\begin{array}{c}\text { Steric } \\
\text { C-18 }\end{array}$ & $\begin{array}{l}\text { Oleic } \\
\text { C-18 } \\
\text { cis } 9 \dagger\end{array}$ & $\begin{array}{c}\text { Linoleic } \\
\mathrm{C}-18 \\
\text { cis, cis } 9,12 \dagger\end{array}$ \\
\hline $0.0 \ddagger$ & 1.00 & 1.00 & 1.00 & 1.00 & 1.00 & 1.00 & 1.00 & 1.00 & 1.00 \\
\hline \multirow[t]{2}{*}{1.0} & 1.09 & 1.09 & 1.16 & 1.77 & 1.32 & 1.19 & 1.34 & 1.36 & 1.46 \\
\hline & $(0.02) \S$ & $(0.01)$ & $(0.01)$ & $(0.01)$ & $(0.04)$ & $(0.02)$ & $(0.03)$ & $(0.01)$ & $(0.01)$ \\
\hline \multirow[t]{2}{*}{2.5} & 1.14 & 1.13 & 1.43 & 2.74 & 2.24 & 2.16 & 2.28 & 2.09 & 2.06 \\
\hline & $(0.02)$ & $(0.02)$ & $(0.01)$ & $(0.02)$ & $(0.02)$ & $(0.13)$ & $(0.03)$ & $(0.02)$ & $(0.06)$ \\
\hline \multirow[t]{2}{*}{5.0} & 1.05 & 1.09 & 2.01 & 3.58 & 3.46 & 3.21 & I & 2.99 & 3.12 \\
\hline & $(0.05)$ & $(0.03)$ & $(0.04)$ & $(0.14)$ & $(0.07)$ & $(0.12)$ & & $(0.02)$ & $(0.01)$ \\
\hline
\end{tabular}

* Values are the amount of lumirubin formed in sample with added fatty acid divided by the amount of lumirubin formed in a simultaneously irradiated reference solution with no fatty acid.

$\dagger$ Position and configuration of double bonds in unsaturated fatty acids.

$\ddagger$ Reference solution containing no added fatty acid.

$\S$ Values in parentheses are the deviation of duplicate experiments.

If Five equivalents of stearic acid would not go into solution.

The binding of fatty acids of carbon chain length of 10 or more to bilirubin-albumin complexes led to a progressive shift in the absorption maximum to longer wavelength (bathochromic shift) and a progressive increase in the strength of the absorption maximum (hyperchromicity). This is illustrated in Figure 1 for the binding of lauric acid to bilirubin-albumin complexes. The binding of the other long-chain fatty acids produced qualitatively similar shifts in the bilirubin absorption spectrum, and all spectra had an isosbestic point at $442 \pm 2 \mathrm{~nm}$. Absorption spectra of samples containing hexanoic or octanoic acid were indistinguishable from the spectrum for bilirubin:albumin alone. Bilirubin contains no asymmetric carbons and is not optically active when dissolved in organic or aqueous solution. However, when bound to albumin, bilirubin has a strong bisignate circular dichroism signal which is the result of the dissymmetric binding of bilirubin in a right-handed helical conformation (11). The binding of the long-chain fatty acids also affected the position and intensities of the circular dichroism bands of bilirubin. This is illustrated in Figure 2 for the binding of lauric acid to bilirubin:albumin complexes. The binding of the other long-chain fatty acids produced qualitatively similar changes in the circular dichroism spectrum of bilirubin. The circular dichroism spectra of samples containing hexanoic or octanoic acid were indistinguishable from the spectrum for bilirubin:albumin alone. Thus, except for hexanoic and octanoic acids, the binding of fatty acids produced changes in the rate of lumirubin formation, the visible absorption spectrum, and the circular dichroism spectrum.

\section{DISCUSSION}

During phototherapy, light energy is used to convert unconjugated bilirubin from an insoluble, unexcretable form to more water-soluble photoproducts. The major photoproducts formed in vivo are configurational isomers, mainly $4 Z, 15 E$-bilirubin, and a structural isomer, lumirubin. In human infants, the more rapidly formed configurational isomers are excreted only very slowly (4). Evidence from a number of clinical studies indicates that the rapid excretion of the more slowly formed lumirubin is the principal route of bilirubin elimination during phototherapy $(2,4,5)$.

Fatty acids bind to human albumin at sites which are distinct from the primary bilirubin binding site (12). The binding of fatty acids has an effect on the circular dichroism spectrum of albumin-bound bilirubin (Fig. 3) which has been reported previously $(9,13)$. This change in the circular dichroism spectrum has been interpreted to be the result of a conformational change of the bilirubin molecule about the central methylene bridge such that the dihedral angle between the two dipyrrolic chromophores of bilirubin is altered (14). This effect of fatty acid binding on the conformation of albumin-bound bilirubin is shown schemati-

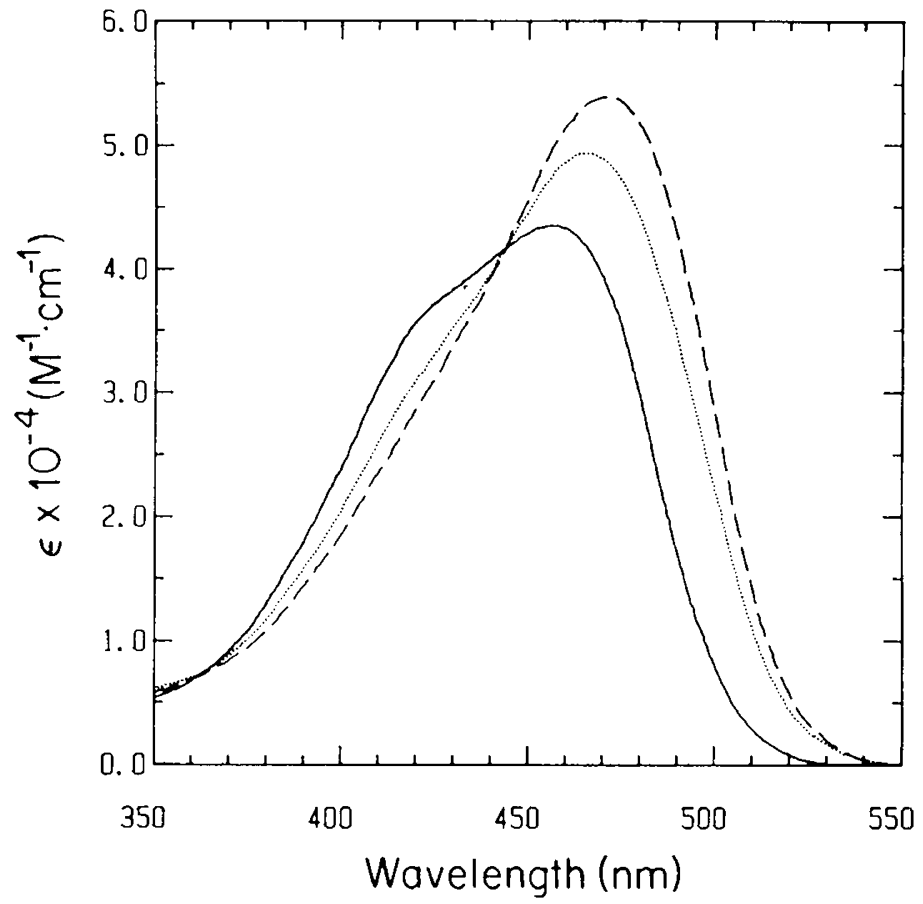

Fig. 1. The effect of lauric acid binding to human albumin on absorption spectrum of bilirubin. The molar extinction coefficient, $\epsilon$, is plotted as a function of wavelength. No lauric acid (solid line); 2.5 equivalents of lauric acid per mole of human albumin (dotted line); 5.0 equivalents of lauric acid per mole of human albumin (dashed line).

cally in Figure 3. Because the photochemistry of bilirubin is sensitive to the surrounding environment, we examined the effect of fatty acid binding on the rate of lumirubin formation. The results in Table 1 demonstrate that those fatty acids which bind to albumin and produce a conformational change in bilirubin enhance the rate of formation of lumirubin.

Two factors contribute to the increased rate of lumirubin formation. One factor is that with the addition of fatty acids, bilirubin bound to albumin absorbs light more strongly (Fig. 1). Therefore, with equal incident irradiation, as used in these experiments, the bilirubin:albumin solutions containing fatty acids will absorb more photons. However, the hyperchromicity produced by fatty acid binding is small when compared to the increased rate of lumirubin production. For example, the increased light absorption in the samples containing 5 equivalents of lauric acid was less than $18 \%$ [estimated by numerical integration of the overlap between the emission spectrum of the light 


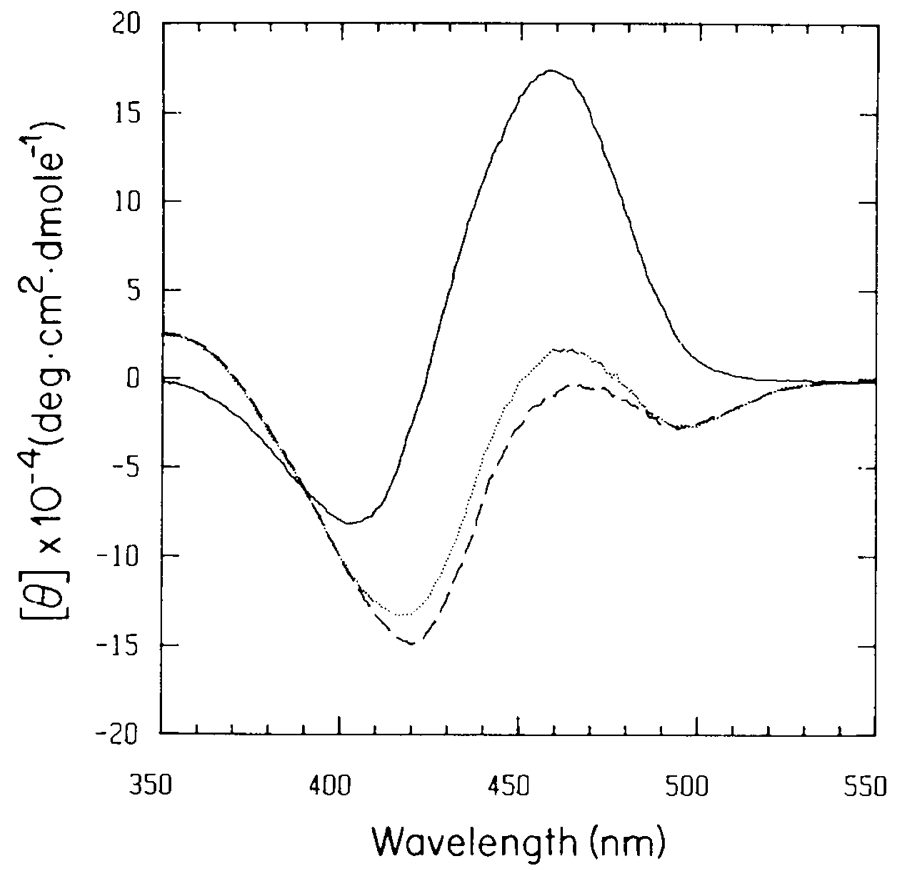

Fig. 2. The effect of lauric acid binding to human albumin on circular dichroism spectrum of bilirubin. Molar ellipticity $(\theta)$ is plotted as a function of wavelength. No lauric acid (solid line); 2.5 equivalents of lauric acid per mole of human albumin (dotted line); 5.0 equivalents of lauric acid per mole of human albumin (dashed line).

source (15) and the absorption spectra for samples containing 0 and 5 equivalents of lauric acid], whereas the increase in lumirubin production is more than $300 \%$. The major contributor to the enhanced production of lumirubin with increasing fatty acid:albumin molar ratio is an increase in the quantum yield for the formation of lumirubin. An albumin-bound bilirubin molecule that absorbs a photon is more likely to form lumirubin if fatty acids are also bound to the albumin. This is presumably the result of the conformational change in the bilirubin molecule produced by the fatty acid binding (Fig. 3), although proof of this relationship will require further work.

Recent studies have provided evidence that during phototherapy the formation of lumirubin is the step which determines the rate at which bilirubin is eliminated $(5,16)$. The importance of lumirubin in the therapeutic efficacy of phototherapy has been somewhat surprising to many investigators because of the very low quantum yield for the formation of lumirubin, especially when compared to the quantum yield for the $Z$ to $E$ configurational isomerization (i.e., 0.0012 versus 0.20) (17) (Malhotra V, Ennever JE, unpublished data). However, all previous in vitro studies of the rate of lumirubin formation have used bilirubin bound to albumin which has been defatted. Our results suggest that the rate of formation of lumirubin in vivo, where the molar ratio of fatty acid:albumin is generally between 0.5 and 3.0 (19), is higher than that predicted based upon in vitro studies with defatted albumin.

Our results suggest that the rate of lumirubin production may be faster and phototherapy perhaps more effective if the fatty acid:albumin ratio were maintained at the higher range of normal. A study is in progress to determine if the rate of formation of lumirubin in jaundiced infants receiving phototherapy can be correlated with fatty acid:albumin molar ratios. It is well known that very high levels of fatty acids can result in the displacement of bilirubin from albumin both in vitro and in vivo (20-23). Thus, the administration of fatty acid emulsions to jaundiced infants is potentially dangerous. The allosteric conformational change in bilirubin bound to albumin which we have associated with an increased rate of lumirubin formation is a more subtle
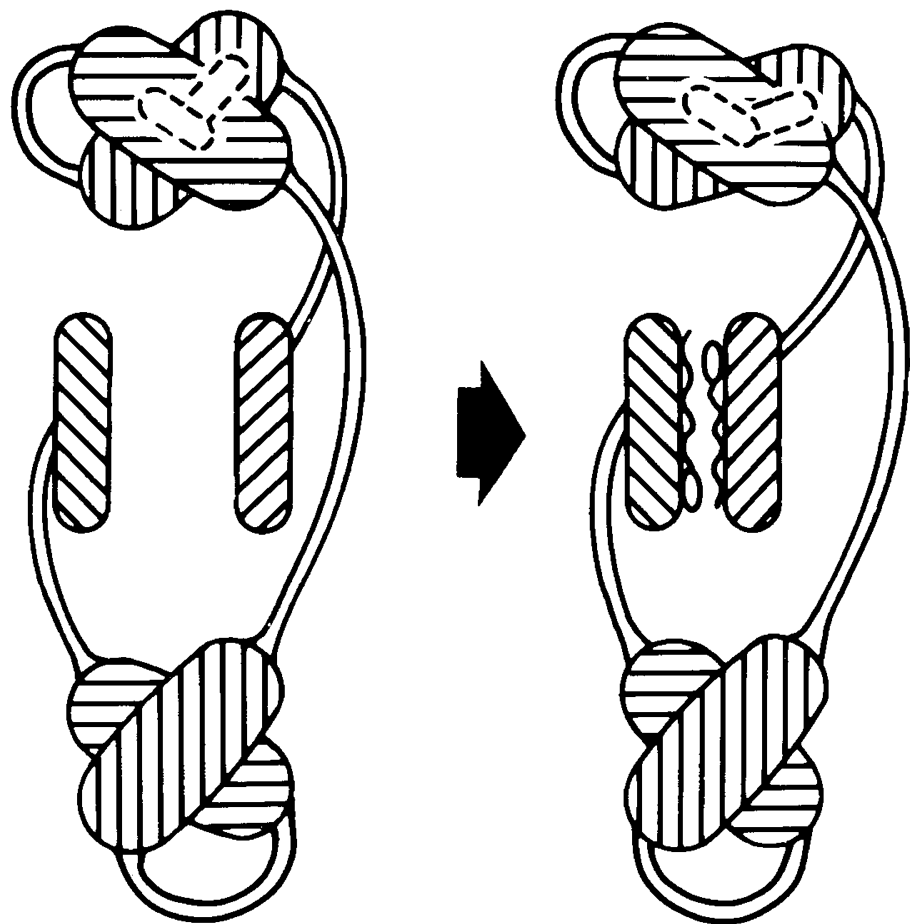

Fig. 3. Schematic representation of the binding of bilirubin to albumin in the absence (left) and presence (right) of fatty acids. The two dipyrrolic chromophores of bilirubin, represented as dashed ovals, are bound to separate domains on albumin, represented as the larger, hatched ovals. In the absence of fatty acids, the angle between the two chromophores is $109^{\circ}(11)$. Fatty acids, represented as an oval hydrophilic end and a long hydrophobic tail, bind to two other domains on albumin, causing a conformational change in the protein and an increase in the dihedral angle between the two chromophores of bilirubin [modified from Brodersen (24)].

change than displacement and occurs at lower fatty acid:albumin molar ratios.

\section{REFERENCES}

1. Cremer RJ, Perryman PW, Richards DH 1958 Influence of light on the hyperbilirubinaemia of infants. Lancet 1:1094-1097

2. Onishi S, Isobe K, Itoh S, Kawade N, Sugiyama S 1980 Demonstration of a geometric isomer of bilirubin-IX $\alpha$ in the serum of a hyperbilirubinaemic newborn infant and the mechanism of jaundice phototherapy. Biochem $\mathbf{J}$ 190:533-536

3. Ennever JF, Speck WT 1984 Mechanism of action of phototherapy: New concepts. In: Rubaltelli R, Jori G (eds) Neonatal Jaundice. Plenum Press, New York, pp 187-196

4. Ennever JF, Knox I, Denne SC, Speck WT 1985 Phototherapy for neonatal jaundice: in vivo clearance of bilirubin photoproducts. Pediatr Res 19:205208

5. Onishi S, Isobe K, Itoh S 1986 Metabolism of bilirubin and its photoisomers in newborn infants during phototherapy. J Biochem 100:789-795

6. Greene BI, Lamola AA, Shank CV 1981 Picosecond primary photoprocesses of bilirubin bound to human serum albumin. Proc Natl Acad Sci USA 78:2008-2012

7. Lamola AA, Flores J 1982 Effect of buffer viscosity on the fluorescence of bilirubin bound to human serum albumin. J Am Chem Soc 104:2530-2534

8. McDonagh AF, Assisi F 1972 The ready isomerization of bilirubin-IX $\alpha$ in aqueous solution. Biochem J 129:797-800

9. Woolley PV, III, Hunter MJ 1970 Binding and circular dichroism data on bilirubin-albumin in the presence of oleate and salicylate. Arch Biochem Biophys 140:197-209

10. Ennever JF, McDonagh AF, Speck WT 1983 Phototherapy for neonatal jaundice: optimal wavelengths of light. J Pediatr 103:295-299

11. Lightner DA, Gawronski JK, Gawronska K 1985 Conformational enantiomerism in bilirubin. Selection by cylodextrins. J Am Chem Soc 107:24562461

12. Berde CB, Hudson BS, Simoni RD, Sklar LA 1979 Human serum albumin Spectroscopic studies of binding and proximity relationships for fatty acids and bilirubin. J Biol Chem 254:391-400

13. Blauer G, Harmatz D 1972 Optical properties of bilirubin-serum albumin complexes in aqueous solution. II. Effects of electrolytes and of concentra- 
tion. Biochim Biophys Acta 278:89-100

14. Blauer $G$, Wagniere $G 1975$ Conformation of bilirubin and biliverdin in their complexes with albumin. J Am Chem Soc 97:1949-1954

15. Ennever JF, Sobel M, McDonagh AF, Speck WT 1984 Phototherapy for neonatal jaundice: in vitro comparison of light sources. Pediatr Res 18:667670

16. Costarino AT, Ennever JF, Baumgart S, Speck WT, Paul M, Polin RA 1985 Bilirubin photoisomerization in premature neonates under low- and highdose phototherapy. Pediatrics 75:519-522

17. Lamola AA, Flores J, Doleiden FH 1982 Quantum yield and equilibrium position of the configurational photoisomerization of bilirubin bound to human serum albumin. Photochem Photobiol 35:649-654

18. Deleted in proof

19. Andrew G, Chan G, Schiff D 1976 Lipid metabolism in the neonate I. The effects of Intralipid infusion on plasma triglyceride and free fatty acid concentrations in the neonate. J Pediatr 88:273-278

20. Thiessen H, Jacobsen J, Brodersen R 1972 Displacement of albumin-bound bilirubin by fatty acids. Acta Paediatr Scand 61:285-288

21. Andrew G, Chan G, Schiff D 1976 Lipid metabolism in the neonate II. The effect of Intralipid on bilirubin binding in vitro and in vivo. $J$ Pediatr 88:279284

22. Odell GB, Cukier JO, Ostrea EM, Maglalang AC, Poland RL 1977 The influence of fatty acids on the binding of bilirubin to albumin. $J$ Lab Clin Med 89:295-307

23. Spear ML, Stahl GE, Paul MH, Engler JM, Pereira GR, Polin RA 1985 The effect of 15-hour fat infusions of varying dosage on bilirubin binding to albumin. JPEN 9:144-147

24. Brodersen R 1982 Physical chemistry of bilirubin: Binding to macromolecules and membranes. In: Heirwegh KPM, Brown SB (eds) Bilirubin, Vol 1. CRC Press, Boca Raton, FL, pp 75-123

\section{Announcement}

\section{Symposium on Developmental Gastroenterology}

The Ohio State University and the Children's Hospital Research Foundation, Columbus, OH will hold the First Wexner Symposium at the Hyatt Regency Hotel from September 13 through September 15, 1987. The symposium has been approved for 10 hours of CME credit.

Invited faculty: Vay Liang W. Go, M.D.; Michael D. Gershon, M.D.; Alan Lucas, M.B., B. Chir, M.R.C.P.; William Balisteri, M.D.; Susan J. Henning, Ph.D.; Otakar Koldovsky, M.D., Ph.D.; Zane Cohen, M.D., F.R.C.S.(C), F.A.C.S.; W. Allan Walker, M.D.

Major topics: bacterial colonization of the gut; gut hormone responses; action of pancreatic polypeptide; signaling and neural messages in the gut; endogenous intestinal hormones; hormones in milk; glucose polymer assimilation; newborn colonic function; intestinal vascular regulation; immune function in the gut; and immunology of intestinal transplantation.

For further information contact: Howard R. Sloan, M.D., Ph.D., Children's Hospital, 205 Ross Hall, 700 Children's Drive, Columbus, $\mathrm{OH} 43205$ or call 614-461-2212. 